NOUVELle

\section{Un polymorphisme fréquent dans le promoteur d'un chimio-attractant des monocytes modifie la susceptibilité à la tuberculose pulmonaire}

Ryad Tamouza, Dominique Labie
R. Tamouza: Laboratoire d'Immunologie

et d'Histocompatibilité, CIB-HOG,

AP-HP/GHU-Nord, IUH et Inserm U662,

Hôpital Saint-Louis,

1, avenue Claude Vellefaux,

75010 Paris, France.

tamouza@histo.chu-stlouis.fr

D. Labie : Département de génétique,

développement et pathologie moléculaire, Institut Cochin,

24, rue du Faubourg Saint-Jacques,

75014 Paris, France.

labie@cochin.inserm.fr

susceptibilité à la TP [8], les auteurs ont montré que les patients porteurs de l'allèle $-2518 \mathrm{G}$ en simple dose avaient 2,3 fois plus de risque de développer une tuberculose active, alors que pour les homozygotes $G G$ le risque était 5,4 fois plus important. Ces données montraient donc un effet dose du polymorphisme A-2518G sur la susceptibilité à la TP. La validité des résultats obtenus sur cette population mexicaine était confortée par le fait que d'autres SNP, non liés à la maladie, étaient observés en équilibre de Hardy-Weinberg.

Dans une seconde étape, ces résultats ont été confirmés dans une population coréenne (malades/témoins: 129/162), ethniquement différente et plus homogène, issue de Mongols et d'Asiatiques du Nord. Dans ce cas, le risque était 2,8 et 6,9 fois plus important chez les individus $A G$ et $G G$ respectivement, confirmant ainsi, et même renforçant les données obtenues au Mexique. Le choix judicieux des catégories de témoins sains (IDR+ et IDR $)$ a permis de préciser que le variant $G$ est significativement associé au passage de l'état de primo-infection (latent) à l'état de tuberculose active et non à la susceptibilité intrinsèque d'être infecté par la bactérie.

Les résultats obtenus apparaissent en désaccord avec ceux d'une étude précédente, effectuée au Brésil, où le variant MCP-1 semblait n'avoir aucun effet sur la susceptibilité à la tuberculose pulmonaire [8]. Cette discordance est probablement liée au choix des populations témoins de la première étude (utilisation de pseudo-témoins familiaux) qui fait perdre de sa puissance au calcul statistique. Elle pourrait être due aussi à un terrain génétique différent. sieurs SNP (single nucleotide polymorphi localisés sur le chromosome 17 (17q11.2), région déjà identifiée comme associé à la

$>$ La tuberculose pulmonaire (TP), due à l'infection par Mycobacterium tuberculosis (MT), est, avec le Sida et le paludisme, une des trois pathologies infectieuses majeures qui sévissent à l'état pandémique. En effet, plus de 8 millions de cas nouveaux et aux alentours de deux millions de décès sont recensés annuellement $[1,2]$. Les études épidémiologiques composante génétique $[3,4]$.

résistants requiert la mise en place d'une réponse immunitaire cellulaire spécifique de sence au site infectieux de cellules spécialisées (macrophages alvéolaires, lymphocytes T CD4 ${ }^{+}$, (IL-12, IL-18 et TNF- $\alpha$ ) ainsi que de chimiokiéraction négative à la tuberculine, d'où Hypoimmunitaire naturelle à l'infection par MT fait aboutir à l'induction d'une réponse spécifique. On sait, par ailleurs, que parmi les individus exposés, même en régions de forte endémie,
seuls $10 \%$ feront une tuberculose active, ce qui permettrait de distinguer la susceptibilité à l'infection de sa progression vers une pathologique, il s'agira dans la moitié des cas, pour les autres, ce sera plutôt une réactivation ou une tuberculose post-primaire qui survient à un âge plus avancé. De nombreuses observa-
tions ont suggéré une prédisposition familiale à la maladie, et, sans doute, l'existence d'une Le contrôle de l'infection chez les individus $\mathrm{CD}^{+}$et $\gamma \delta$ ), de cytokines pro-inflammatoires
\end{abstract}

Mestizos, du district fédéral de Mexic sont sur le plan ethnique issus d'un mélange entre Hispaniques, Amérindiens et Noirs africains. En étudiant la distribution de plu-

nes (RANTES, MIP- $1 \alpha$, IL-8 et MCP- 1 ), dont l'action sur la migration des différentes site est déterminante pour la formation du granulome [5]. II est admis, en ce qui concerne cette dernière catégorie, que MCP-1 (monocyte chemoattractant protéine-1), un puissant «chimio-attractant 》 des pes lymphocytes $\mathrm{T}$, occ Une recherche pluricentrique récente a été menée d'abord sur une population mexi[7]. Dans l'exploration des sujets mexicains, les auteurs ont étudié 445 patients récemment infectés qu'ils ont comparés à 518 témoins sains, dont 334 étaient positifs à l'intradermoréaction à la tuberculine (IDR $\left.{ }^{+}\right)$ et 176 négatifs $\left(\mathrm{IDR}^{-}\right)$. Des signes radiologiques de tuberculose pulmonaire étaient promoteur du gène $M C P$-1, en l'occurrence la mutation $-2518 \mathrm{~A} \rightarrow \mathrm{G}$, était un facteur de risque hautement associé au passage de la forme latente à la forme active de la TP l'objet de cette étude est une population panmictique, composée pour l'essentiel de 
Cette dernière hypothèse paraît, cependant, moins probable, étant donné que les résultats obtenus au Mexique ont été confirmés sur une seconde population génétiquement différente. Ayant constaté que le taux de MCP-l était plus élevé chez les patients tuberculeux que chez les témoins IDR ${ }^{+}$et IDR ${ }^{-}(p=0,00001)$ (Figure $1 A)$, les auteurs ont recherché une corrélation avec l'allèle $\mathrm{G}$. Cette corrélation est extrêmement nette, le taux le plus élevé étant observé chez les sujets porteurs du génotype GG (Figure 1B). Cette augmentation va de pair avec la diminution du taux d'IL-12p40 circulante, un composant nécessaire à l'expression de l'IL-12 (Figure 1B). Cette corrélation négative, hau- tement significative $(p=0,00001)$ suggère que la surproduction de MCP-1 exerce une régulation négative sur l'expression de I'IL-12p40 et donc de l'IL-12.

Les auteurs ont aussi procédé à une vérification expérimentale. Ils ont montré que la stimulation de monocytes de porteurs sains (IDR ${ }^{+}$) et de génotype GG aboutit aux mêmes profils d'expression du tandem MCP-1/IL-12p40. De plus, l'utilisation d'anticorps anti-MCP-1 libère la production d'IL-12 dans le surnageant de culture de monocytes $G G$, alors que l'addition de MCP-1 dans le milieu de culture de monocytes $A A$, induit cette fois une dimi-

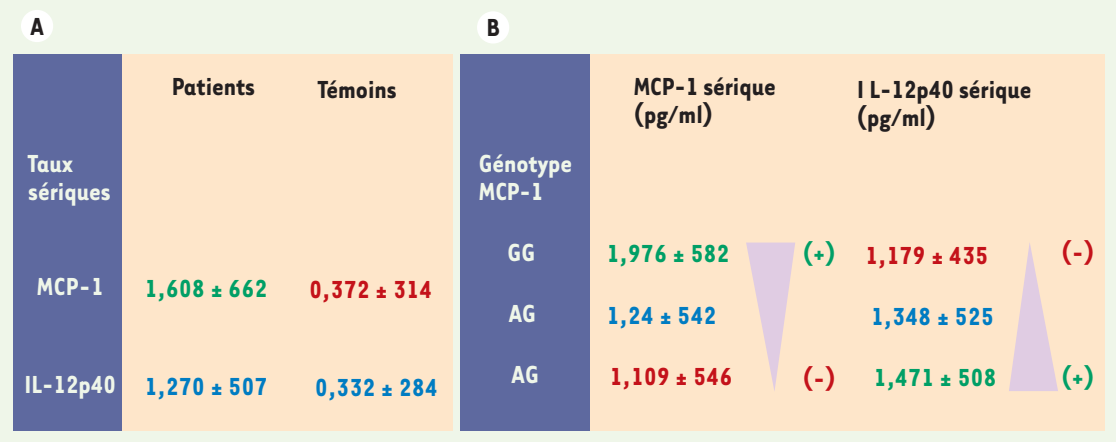

Figure 1. A. Comparaison des taux de MCP-1 et d'IL-12p40 chez les patients tuberculeux et chez les témoins sains $\left(I D R^{+}\right.$et IDR ${ }^{-}$). Les patients montrent un taux significativement plus élevé de $M C P-1$ $(p=0,00001)$. B. Analyse des taux de MCP-1 et d'IL-12p40 en fonction du génotype des patients (MCP1-2518 AA, $A G, G G)$. II existe une corrélation hautement significative avec l'allèle $G$. Le taux le plus élevé de MCP-1 étant observé chez les sujets porteurs du génotype GG. Cette augmentation va de pair avec la diminution du taux d'IL-12p40 circulante $(p=0,00001)$ chez les mêmes patients.

\section{$1^{\text {er }}$ Congrès de la Fondation pour la Recherche ELA}

\section{Rôle vital du développement de la myéline et de sa stabilité : ciblage dans les leucomalacies périventriculaires et les leucodystrophies}

\section{5 -7 octobre 2006 \\ Hôtel Concorde Lafayette - 75017 Paris, France}

Aspects normaux et pathologiques de la myélinisation

Mécanismes conduisant à une destruction des cellules gliales et des axones

Leucomalacies périventricualires chez le prématuré

Maladies héréditaires et la myéline dues à un dysfonctionnement de l'astrocyte

Approches pharmacologiques, de thérapie cellulaire et génique de ces maladies

Renseignements et inscriptions

http://www.ela-fondation.com. nution de production d'IL-12p40. L'ensemble de ces résultats suggère qu'un taux de MCP-1 au-dessus d'un certain niveau exercerait un effet de rétrocontrôle négatif sur la synthèse d'IL-12, ce qui aurait pour effet de déréguler la réponse de la voie Thl.

Il est sûrement intéressant de noter que si ce génotype paraît agir sur la progression de l'infection, il ne semble pas augmenter la susceptibilité à la mycobactérie, permettant ainsi de distinguer les deux processus. Cet effet ayant été retrouvé dans deux populations totalement indépendantes démontre bien l'existence d'une composante génétique dans le développement de la maladie. On ne peut pas, cependant, écarter totalement l'existence de variantes entre diverses populations. Le rôle pivot joué par $\mathrm{IL}-12$ dans la résistance aux pathogènes intracellulaires, dont $M$. tuberculosis, plaide en faveur du mécanisme MCP1/IL-12. Cependant, au vu de sa fréquence élevée dans la population générale, il paraît peu probable que le variant $A 2518 \mathrm{G}$ soit le seul déterminant génétique de la susceptibilité à la tuberculose, mais il est indéniablement un des plus fortement associé. $\nabla$

A promotor polymorphism

in monocyte chemoattractant

is associated with increased

susceptibility to pulmonary tuberculosis

\section{RÉFÉRENCES}

1. Casanova JL, Abel L. Genetic dissection of immunity to mycobacteria : the human model. Annu Rev Immunol 2002 ; $20: 581-620$.

2. Alcais A, Fieschi C, Abel L, Casanova JL. Tuberculosis in children and adults : two distinct genetic diseases. J Exp Med $2005 ; 202$ : 1617-21.

3. Bellamy R, Beyers N, McAdam KP, et al. Genetic susceptibility to tuberculosis in Africans: a genome-wide scan. Proc Natl Acad Sci USA 2000 ; 97 : 8005-9.

4. Bellamy R, Ruwende C, Corrah T, et al. Variations in the NRAMPI gene and susceptibility to tuberculosis in West Africans. N Engl J Med 1998 ; 338 : 640-4.

5. Salgame P. Host innate and Thl responses and the bacterial factors that control Mycobacterium tuberculosis infection. Curr Opin Immunol $2005 ; 17: 374-80$.

6. Lin Y, Gong J, Zhang M, Xue W, Barnes PF. Production of monocyte chemoattractant protein 1 in tuberculosis patients. Infect Immun 1998; 66: 2319-22.

7. Flores-Villanueva PO, Ruiz-Morales JA, Song CH, et al. A functional promoter polymorphism in monocyte chemoattractant protein-l is associated with increased susceptibility to pulmonary tuberculosis. J Exp Med 2005; $202: 1649-58$.

8. Jamieson SE, Miller EN, Black GF, et al. Evidence for a cluster of genes on chromosome 17q11-q21 controlling susceptibility to tuberculosis and leprosy in Brazilians. Genes Immun 2004 ; 5 : 46-57. 